\title{
Dynamics of hexagonal patterns in a self-focusing Kerr cavity
}

\author{
Damià Gomila* and Pere Colet ${ }^{\dagger, *}$ \\ Instituto de Física Interdisciplinar y Systemas Complejos (IFISC, CSIC-UIB), Campus Universitat Illes Balears, \\ E-07122 Palma de Mallorca, Spain
}

(Received 2 February 2007; published 25 July 2007)

\begin{abstract}
In this paper we analyze in detail the secondary bifurcations of stationary hexagonal patterns in a prototype model of nonlinear optics. Hexagonal pattern solutions with all allowed wave numbers are computed and their linear stability is studied by means of a Bloch analysis. Depending on the wave number of the selected pattern we predict and numerically observe phase instabilities, amplitude instabilities, both stationary and oscillatory, and oscillatory finite wavelength bifurcations. The results presented here illustrate a typical bifurcation scenario for patterns with different wave numbers in self-focusing systems.
\end{abstract}

DOI: 10.1103/PhysRevE.76.016217

PACS number(s): 89.75.Kd, 42.65.Sf, 47.54.-r, 47.20.Ky

\section{INTRODUCTION}

The mean field equation describing a Kerr medium in a cavity with flat mirrors and driven by a coherent plane wave is one of the simplest models showing spatial structures in nonlinear optics. This prototype model has been used to study a wide variety of phenomena such as pattern formation in optical bistability or formation of localized structures [1-7]. In the self-focusing case, a weakly nonlinear analysis shows that, for pump intensities above a certain threshold, the system spontaneously develops hexagonal patterns while stripes are unstable [3,8].

Once a hexagonal pattern is formed, increasing further the intensity of the pump, it may undergo secondary instabilities. Secondary bifurcations of hexagonal patterns have been observed experimentally, not only in optics [9-13], but also in hydrodynamical systems [14-18] and gas discharges [19]. From a theoretical point of view, they have been studied mainly by means of amplitude equations or symmetry-based approaches [20-27]. In the first case, amplitude equations are only valid close to the primary instability leading to the formation of the pattern, therefore, only secondary bifurcations occurring close to this point can be analyzed. The second technique classifies the different ways in which the spatial symmetry can be broken, however, does not predict which instability will occur in a particular model. It is then interesting to study in more detail secondary bifurcations of hexagonal patterns in specific systems. In this paper we perform a linear stability analysis of hexagonal pattern solutions in the mean field model for a Kerr cavity. This simple model allowed us to study generic instabilities associated to the self-focusing effect, a commonplace phenomenon in different fields of physics such us nonlinear optics [28,29], fluid dynamics [30], and plasma physics [31].

Secondary instabilities in this model were partially studied in [5] as part of a transition from stationary hexagonal pattern to a regime of spatiotemporal chaos, however, in that case, only a pattern with a precise wave number was analyzed. Here we study the secondary bifurcations of patterns

\footnotetext{
*damia@imedea.uib.es

†pere@imedea.uib.es
}

with different wave numbers and analyze the role of the spatial wavelength in the instabilities. A similar analysis was done in [32,33] for two different systems: an alkali metal vapor in a single-mirror arrangement and a saturable absorber in a cavity. In these two cases, however, only stationary instabilities were found.

This paper is organized as follows: In Sec. II we describe the model and perform a linear stability analysis of the homogeneous solution. In Sec. III we find the stationary hexagonal pattern solutions of the model with any wave number. Then, in Sec. IV, we perform a linear stability analysis of the hexagonal patterns and discuss the different instabilities depending on the wave number of the pattern. Finally, in Sec. $\mathrm{V}$ we give some concluding remarks.

\section{MODEL}

In the mean field approximation, the dynamics of the electric field inside an optical cavity filled with a self-focusing Kerr medium can be described by an equation for the scaled slowly varying amplitude of the field $E(\vec{x}, t)[1,2]$ as follows:

$$
\partial_{t} E=-(1+i \theta) E+i \nabla^{2} E+E_{0}+i 2|E|^{2} E,
$$

where $E_{0}$ is the input field, $\theta$ is the cavity detuning, and $\nabla^{2}$ is the transverse Laplacian. Equation (1) has been normalized by the cavity decay rate. The homogeneous solution $E_{s}$ of Eq. (1) is implicitly given by $E_{0}=E_{s}\left[1-i\left(2 I_{s}-\theta\right)\right]$, where $I_{s}$ $\equiv\left|E_{s}\right|^{2}$. The relation between the input and output optical intensities is then given by

$$
I_{0}=I_{s}\left[1+\left(2 I_{s}-\theta\right)^{2}\right],
$$

where $I_{0} \equiv\left|E_{0}\right|^{2}$. Through this paper we consider $\theta<\sqrt{3}$ so that from Eq. (2) it follows that there is only one homogeneous solution. Then $I_{s}$, directly related to $I_{0}$ via Eq. (2), can be considered as a proper control parameter.

A linear stability analysis of the homogeneous solution with respect to spatially periodic perturbations yields to the dispersion relation

$$
\lambda(\vec{k})=-1 \pm \sqrt{-\left(\theta+k^{2}-6 I_{s}\right)\left(\theta+k^{2}-2 I_{s}\right)},
$$

where $\lambda(\vec{k})$ is the linear growth rate of a perturbation with wave vector $\vec{k}$ and $k=|\vec{k}|$. 
A pattern forming instability takes place at $I_{s}=I_{t h}=1 / 2$ with a critical wave number $k_{c}=\sqrt{2-\theta}$. For pump intensities above threshold the maximum linear growth rate is for wave vectors with modulus

$$
k_{u}=\sqrt{4 I_{s}-\theta} .
$$

At threshold $k_{u}=k_{c}$. The selection of the wave number $k_{u}$ [which as follows from Eqs. (4) and (2) depends on the pump intensity $I_{0}$ ] arises from a balance between the diffractive phase modulation $k_{u}^{2}$, the nonlinear phase modulation $4 I_{s}$, and the cavity detuning $\theta$. Exact compensation among these three effects, expressed by Eq. (4), results in a perfect phase matching of the four-wave mixing interaction (annihilation of two photons of the steady uniform solution and creation of one pair of photons with opposite transverse wave number $k_{u}$ ) that enhances the perturbation, and hence a maximum growth rate.

\section{STATIONARY HEXAGONAL PATTERNS}

The linear stability analysis gives information about where the homogeneous solution becomes unstable to small perturbations, but not about the nontrivial solution that may emerge from the bifurcation. In two transverse dimensions, a nonlinear analysis predicts the formation of hexagonal patterns $[2,3]$. This is a general feature related to the existence of quadratic nonlinearities in the equation for the dynamics of the perturbations of the homogeneous solution.

Depending on the value of $I_{s}$, the hexagons may appear oscillating. The transition is subcritical and the hexagonal pattern, once it is formed, is stable for a range of values of the pump intensity including values below threshold. In the case of oscillating hexagons, the amplitude of the oscillations decreases when the pump intensity is decreased adiabatically until they become, in all cases, stationary. Due to the subcriticality of the hexagonal patterns, they always have, even at threshold, a finite amplitude. Then, the harmonics of the six fundamental wave vectors have a significant intensity and they have to be considered. This is particularly important in the self-focusing case considered here because the sharp peaks of the pattern have a strongly anharmonic far field (Fig. 1). form

The stationary hexagonal patterns can be written in the

$$
E_{h}(\vec{x})=\sum_{n=0}^{N-1} a_{n} e^{i k_{n}^{0} \vec{x}},
$$

where $\vec{k}_{n}^{0}$ are the wave vectors of the pattern, $a_{n}$ are complex coefficients, and $N=91$ is the number of Fourier modes we consider in the analysis. $\vec{k}_{0}^{0}=0$ is the homogeneous mode and $\vec{k}_{n}^{0}$ for $n=1, N-1$ are the off-axis wave vectors of the hexagonal pattern, containing the six fundamental wave vectors with modulus $\left|\vec{k}_{n}^{0}\right|=k(n=1,6)$ and harmonics up to $5 k$. As the hexagonal patterns are the result of a spontaneous breaking of the translational invariance, there are continuous families of such patterns. Each family is characterized by the pattern wave number $k$ and each member of the family by the
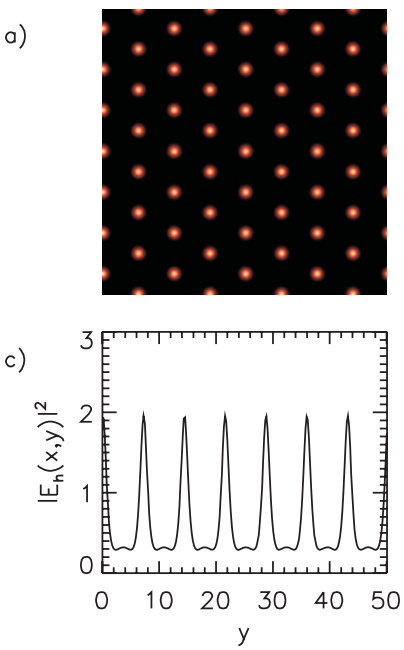

b)

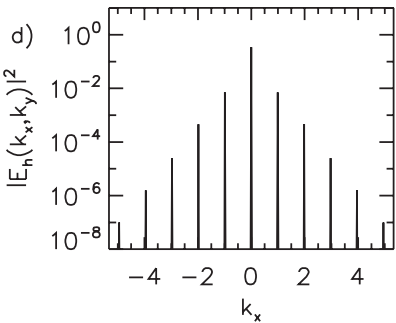

FIG. 1. (Color online) (a) Near field $|E(x, y)|^{2}$ and (b) power spectrum (far field) $|E(\vec{k})|^{2}$ of a stationary hexagonal solution. Note the presence of high harmonics in the far field. Panel (c) shows a cross section along a $y$ axis of the pattern and panel (d) a cross section along the $k_{x}$ axis of the power spectrum. The hexagon drawn in a solid line in panel (b) shows the first Brillouin zone of the lattice defined by the Fourier modes of the hexagonal pattern in the far field.

location of the pattern in the near field, which corresponds to a global phase in the far field.

The coefficients $a_{n}$ can be obtained either by numerical simulations or by solving the stationary form of Eq. (1),

$$
-(1+i \theta) E+i \nabla^{2} E+E_{0}+i 2|E|^{2} E=0 .
$$

The second method allows us to find the stationary hexagonal solution with very high accuracy much faster than from numerical simulations. It also allows us to find both stable and unstable stationary solutions. Substituting Eq. (5) in Eq. (6) a set of coupled nonlinear equations for the amplitudes of the Fourier components $a_{n}$ is obtained

$$
\left[-(1+i \theta)-i\left|\vec{k}_{n}^{0}\right|^{2}\right] a_{n}+i 2 \sum_{l=0}^{N-1} \sum_{m=0}^{N-1} a_{l} a_{m}^{*} a_{n-l+m}=0,
$$

where $a_{n-l+m}$ is the amplitude of the mode $\vec{k}_{n}^{0}-\vec{k}_{l}^{0}+\vec{k}_{m}^{0}$. From a suitably chosen initial condition a Newton-Raphson method is used to find solutions of the set of nonlinear equations (7). The first guess can be obtained from a numerical simulation of Eq. (1). Once a single stationary solution has been found, any parameter region can be reached by means of continuation techniques [34]. We also treat the wave number $k$ of the hexagonal pattern as a parameter and look for stable and unstable stationary hexagonal solutions with different fundamental wave numbers.

Figure 2 shows the region of existence of stationary hexagons. For each value of $I_{s}$ above threshold there is a whole band of possible stationary hexagonal patterns with very different wave numbers $k$. The hexagons are subcritical and therefore exist below the marginal stability curve of the homogeneous solution (black solid line). The lower limit for the existence of a subcritical hexagonal pattern with a given 


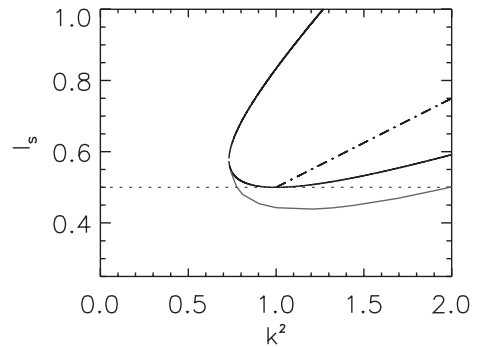

FIG. 2. Marginal stability curve of the homogeneous solution (solid black line), most unstable wave number (dashed line), and the lower limit (saddle-node bifurcation) for the existence of stationary hexagonal patterns (gray line) for $\theta=1.0$.

$k$ (gray solid line) is determined by the saddle-node bifurcation where this pattern branch collides with the branch of unstable hexagonal patterns with the same wave number that starts from the homogeneous solution at the marginal stability curve $\lambda(k)=0$. The upper branch of stationary hexagonal patterns ends at the upper line of the marginal stability curve. These hexagonal patterns, however, become first unstable leading to different dynamical regimes as will be shown in the next section.

\section{SECONDARY BIFURCATIONS OF STATIONARY HEXAGONAL PATTERNS}

In this section we perform a numerical linear stability analysis of the stationary hexagonal solutions with different fundamental wave numbers $k$ in order to determine their stability as a function of the input power [5,32,33]. Linearizing Eq. (1) around the stationary solution (5) the following equation for the perturbations $\delta E(\vec{x}, t)=E(\vec{x}, t)-E_{h}(\vec{x})$ is obtained:

$$
\partial_{t} \delta E=-(1+i \theta) \delta E+i \nabla^{2} \delta E+i 2\left[2\left|E_{h}\right|^{2} \delta E+E_{h} E_{h} \delta E^{*}\right] .
$$

The solution of this equation can be written as a superposition of Bloch waves $[5,32,33]$

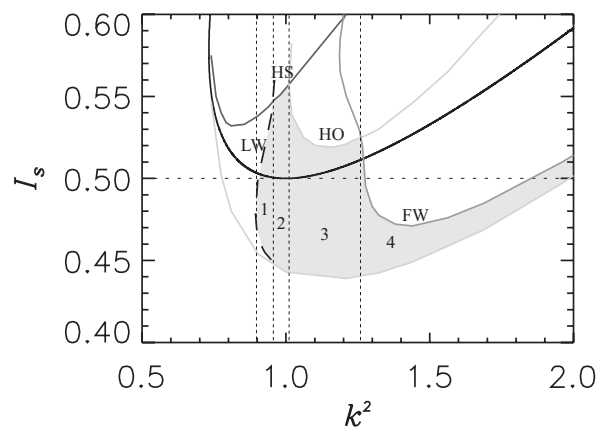

FIG. 3. Marginal stability diagram of the hexagonal pattern solutions for $\theta=1.0$. The shadowed region corresponds to the parameter values where the stationary hexagonal patterns are stable. Each numerated subregion corresponds to hexagonal patterns that undergo different instabilities when the pump is set out of the stable region.
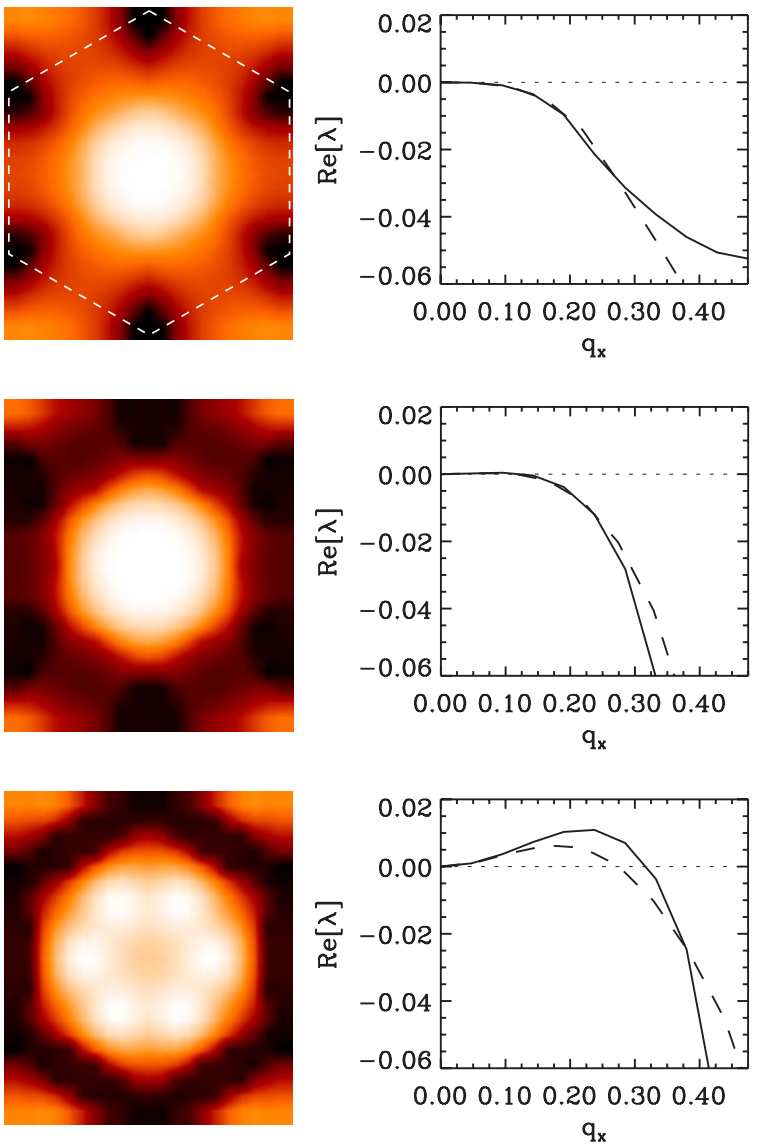

FIG. 4. (Color online) Left: $\operatorname{Re}\left[\lambda_{0}\left(q_{x}, q_{y}\right)\right]$ for a hexagonal pattern with a wave number inside the region $1(k=0.95) . q_{x}=q_{y}=0$ (center of the image) corresponds to the Goldstone modes and $\lambda_{0,1}(\vec{q}=0)=0$. The dashed line shows the limits of the first Brillouin zone. Dark (bright) color indicates low (large) values of $\operatorname{Re}\left[\lambda_{0}\left(q_{x}, q_{y}\right)\right]$. From top to bottom, $I_{s}=0.48$ (stable), $I_{s}=0.50$ (critical point), and $I_{s}=0.52$ (unstable). Right: transverse cut of $\operatorname{Re}\left[\lambda_{0}\left(q_{x}, q_{y}\right)\right]$ along the $q_{x}$ (solid line) and $q_{y}$ (dashed line) axis.

$$
\delta E(\vec{x}, t)=\sum_{n=0}^{N-1}\left[\delta a_{n}(+\vec{q}, t) e^{i\left(\vec{k}_{n}^{0}+\vec{q}\right) x}+\delta a_{n}(-\vec{q}, t) e^{i\left(\vec{k}_{n}^{0}-\vec{q}\right) x}\right] .
$$

A set of linear equations for the complex amplitudes $\delta a_{n}(\vec{q}, t)$ is then obtained from Eq. (8),

$$
\begin{aligned}
\partial_{t} \delta a_{n}( \pm \vec{q})= & {\left[-(1+i \theta)-i\left|\vec{k}_{n}^{0} \pm \vec{q}\right|^{2}\right] \delta a_{n}( \pm \vec{q}) } \\
& +i 2\left[2 \sum_{l=0}^{N-1} \sum_{m=0}^{N-1} a_{l} a_{m}^{*} \delta a_{n-l+m}( \pm \vec{q})\right. \\
& \left.+\sum_{l=0}^{N-1} \sum_{m=0}^{N-1} a_{l} a_{m} \delta a_{-n+l+m}^{*}(\mp \vec{q})\right] .
\end{aligned}
$$

By considering $\vec{\Sigma}(\vec{q}, t)=\left(\delta a_{1}(\vec{q}, t), \delta a_{2}(\vec{q}, t), \ldots, \delta a_{N}(\vec{q}, t)\right.$, $\left.\delta a_{1}^{*}(-\vec{q}, t), \ldots, \delta a_{N}^{*}(-\vec{q}, t)\right)^{T}$, Eq. (9) can be written as

$$
\partial_{t} \vec{\Sigma}(\vec{q}, t)=M\left(a_{n}, \vec{q}\right) \vec{\Sigma}(\vec{q}, t) .
$$

Then, we find the $2 N$ eigenvalues $\lambda_{n}(\vec{q})(n=0, \ldots, 2 N-1)$ of the matrix $M\left(a_{n}, \vec{q}\right)$, and its corresponding eigenvectors, for 

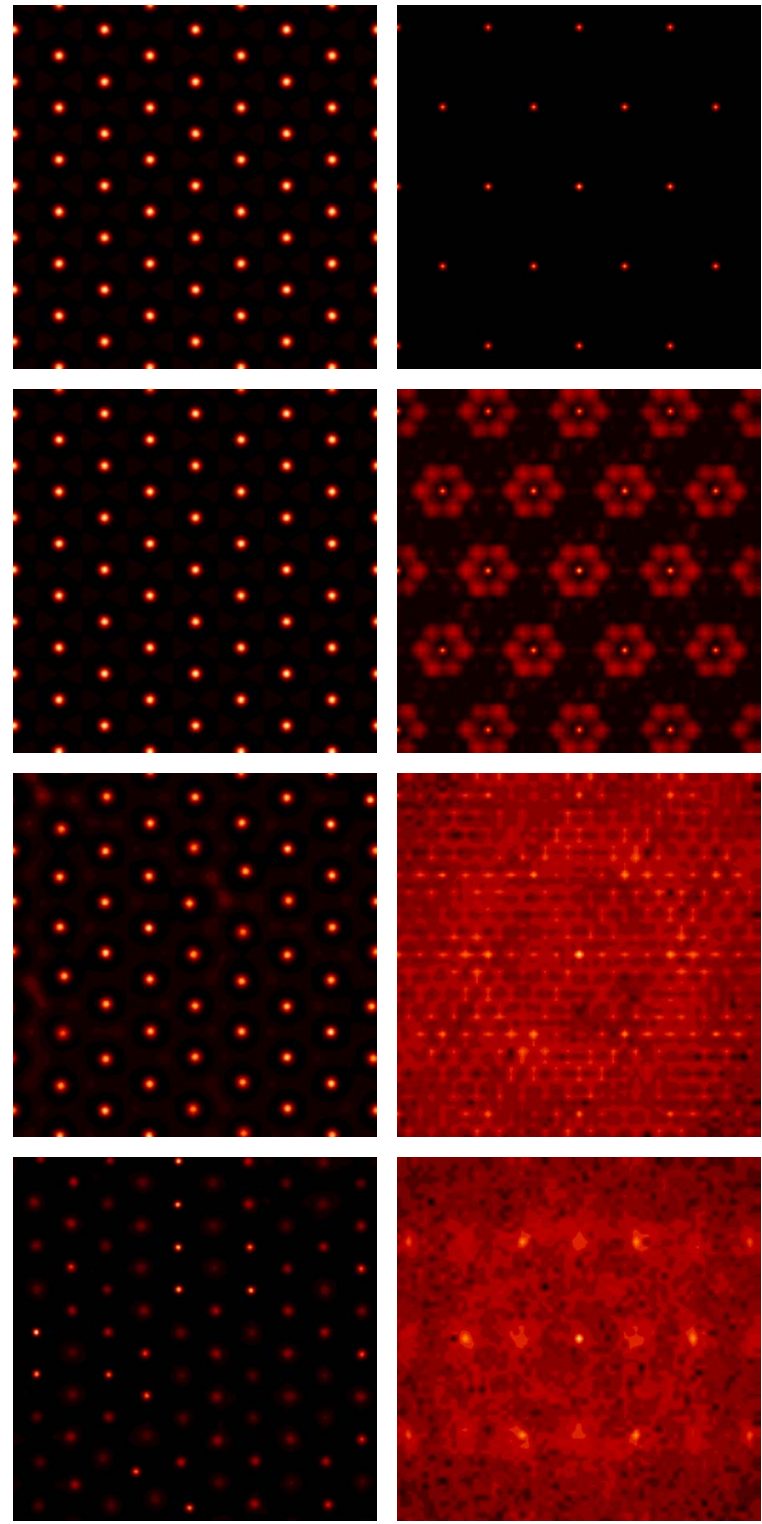

FIG. 5. (Color online) Time evolution of the near (left) and far field (right) of an unstable hexagonal pattern. The wave number of the initially unstable hexagons is $k=0.95$ and $I_{s}=0.52$. The time increases from top to bottom. The right pictures show the central part of the far field in the range $k_{x}=[-2 k, 2 k]$ and $k_{y}$ $=[-16 k / 7,16 k / 7]$.

each vector $\vec{q}$. These eigenvalues may be either real or complex conjugates and determine the stability of the solution against periodic perturbations containing any set of wave vectors $\vec{k}_{n}^{0} \pm \vec{q}$. It is sufficient to explore $1 / 6$ of the first Brillouin zone [35] plotted in Fig. 1(b). We order the eigenvalues $\lambda_{i}(\vec{q})$ according to the values of their real part $\operatorname{Re}\left[\lambda_{i}(\vec{q})\right]$ $\geqslant \operatorname{Re}\left[\lambda_{i+1}(\vec{q})\right]$

We find that the stationary hexagonal patterns are stable only in the shadowed region of Fig. 3. Lines LW (long wavelength), HS (homogeneous stationary), HO (homogeneous oscillatory), and FW (finite wavelength) indicate different types of instabilities. Increasing or decreasing the pump intensity stable hexagonal patterns become unstable in differ-
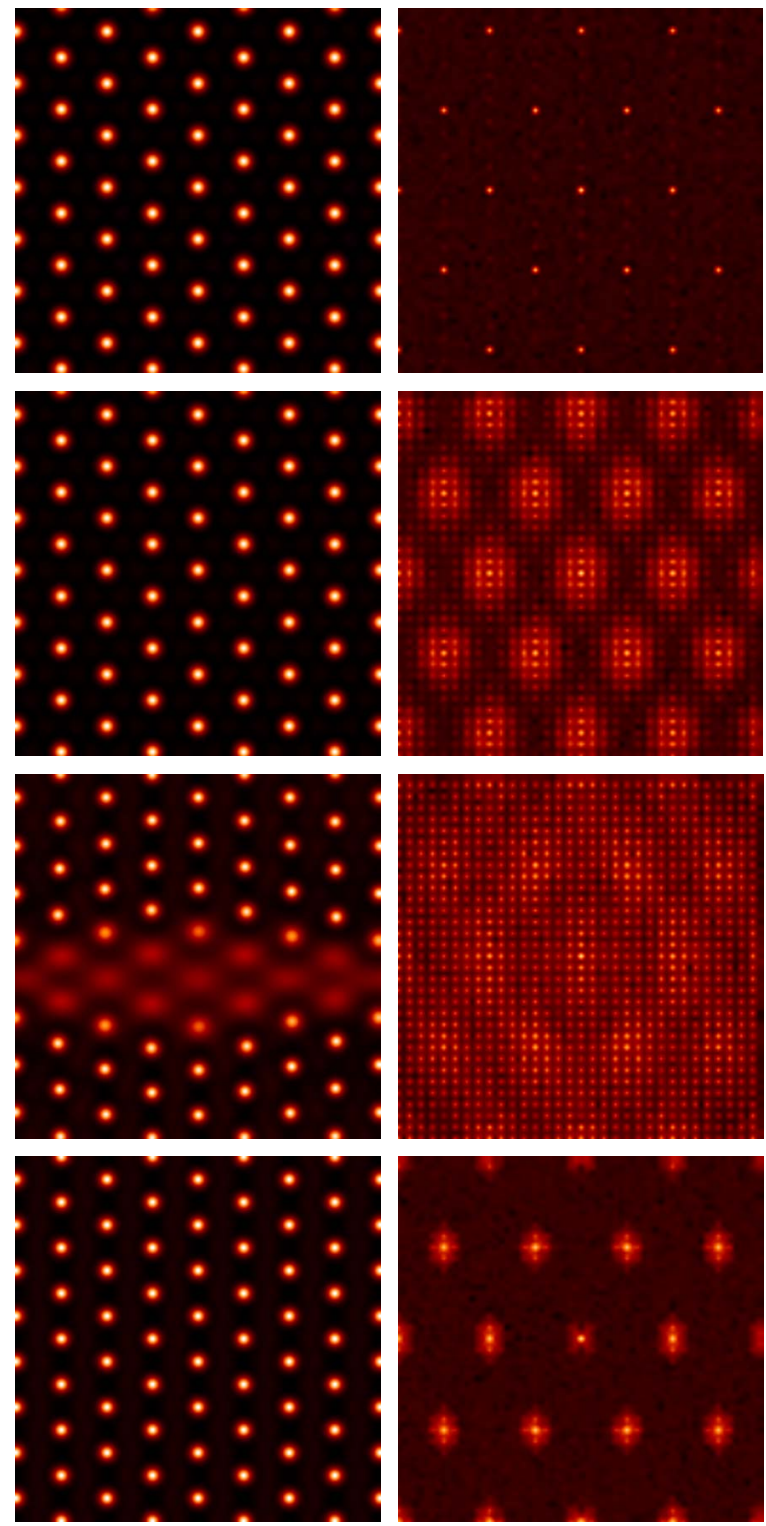

FIG. 6. (Color online) The same as in Fig. 5 for $I_{s}=0.46$. In this case the final state is a stationary hexagonal pattern with one more spot in the $y$ direction.

ent ways depending on their wave number. In the following subsections we analyze the different cases. Numerical simulations have been performed on a $512 \times 512$ rectangular grid with periodic boundary conditions. For a stationary hexagonal pattern of wave number $k$ we use $\Delta k_{x}=k / 8$ and $\Delta k_{y}$ $=k \sin \left(60^{\circ}\right) / 7$ such that the Fourier space in the modes lie exactly on top of the grid. This discretization allows for higher order harmonics (up to 32) in Fourier space ensuring that any relevant wavelength is properly accounted for. In real space, the system size corresponds to $L_{x}=16 \pi / k$ and $L_{y}=28 \pi /(\sqrt{3} k)$. This size is large enough to account for a proper observation of the predicted instabilities.

\section{A. Long-wavelength instability}

Hexagons with small wave numbers (region 1 in Fig. 3) become unstable against long-wavelength perturbations $(\vec{q}$ 

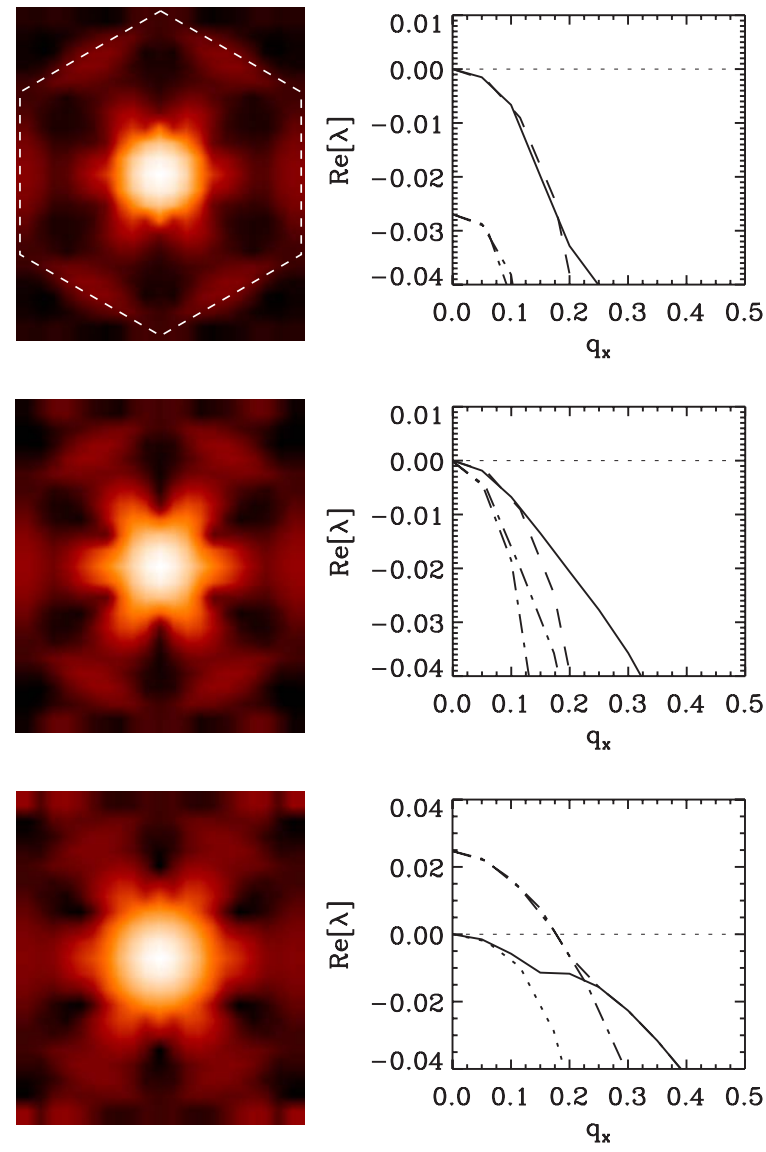

FIG. 7. (Color online) The same as in Fig. 4 for hexagons with a wave number $k=1.0$ crossing the upper border of region 2. From top to bottom $I_{s}=0.55$ (stable), $I_{s}=0.555$ (critical point), and $I_{s}$ $=0.56$ (unstable). Note that the branch that becomes unstable (dotted-dashed lines) is not the branch of the Goldstone and soft modes (solid and dashed lines).

$\sim 0$ ) when the pump is changed such that $I_{s}$ goes across the dashed border (LW). This instability is associated to the dynamics of the global phase, or position, of the pattern [36], and is typically called phase instability (see, e.g., [33,34,37-39] for previous work on phase instabilities of hexagons). For small $|\vec{q}|$ the branches of eigenvalues $\lambda_{0,1}(\vec{q})$ have a parabolic shape $\operatorname{Re}\left[\lambda_{0,1}(\vec{q})\right] \propto|\vec{q}|^{2}$, and this instability is characterized by a change of their convexity. These branches correspond to the so-called soft modes associated to
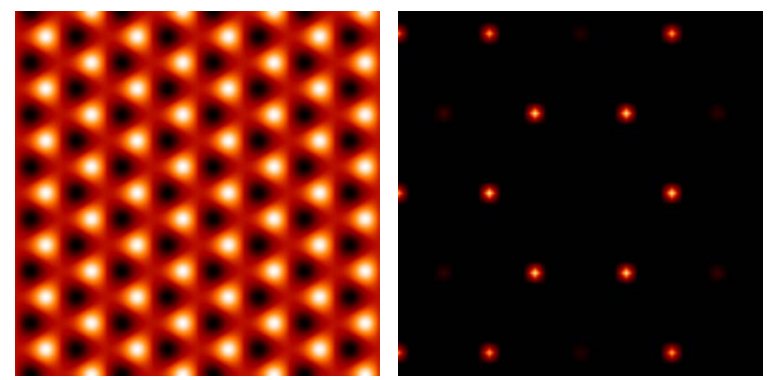

FIG. 8. (Color online) Real part of the near field and far-field intensity of the mode then become unstable when the upper border of region 2 is crossed.
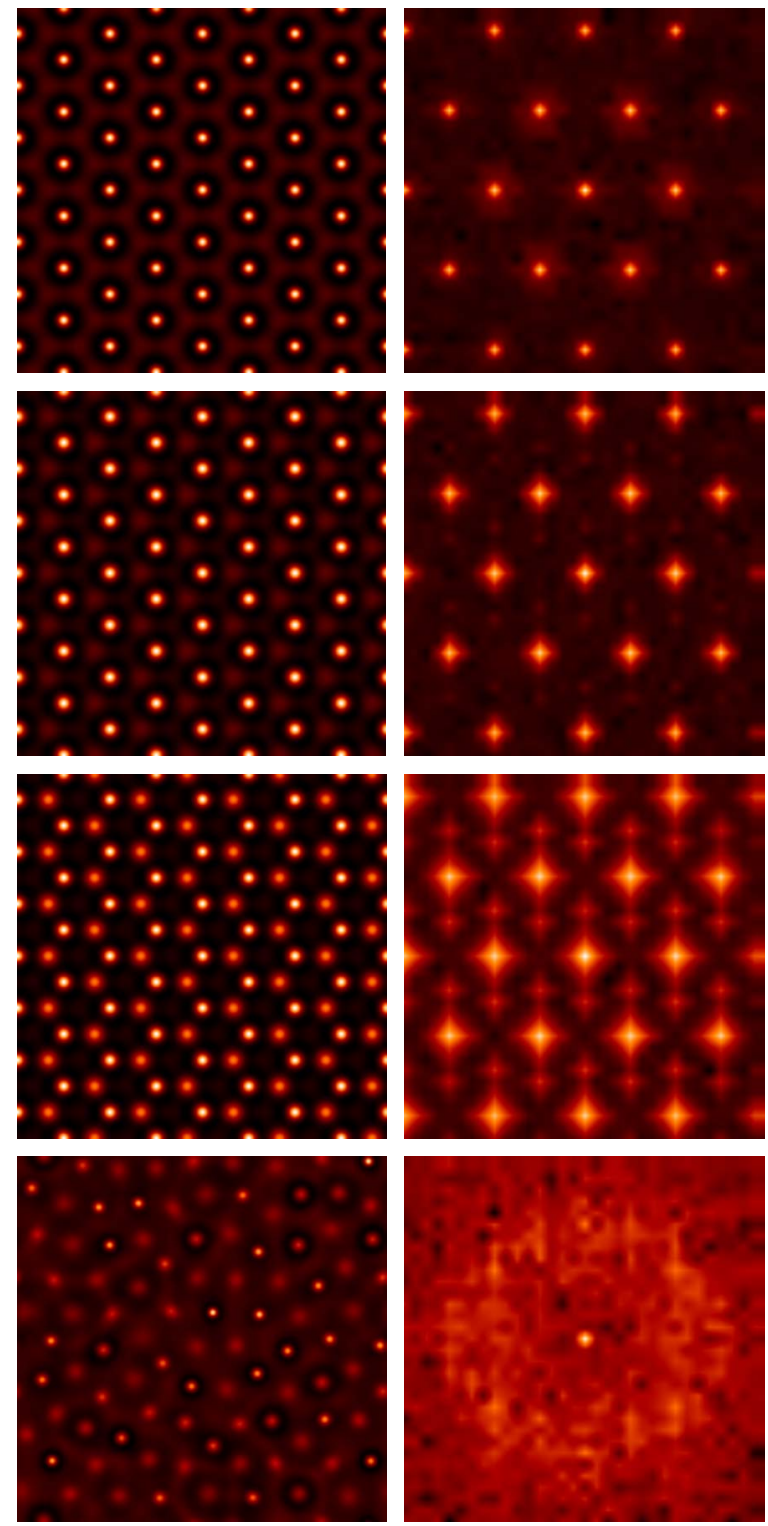

FIG. 9. (Color online) The same as in Fig. 5 for unstable hexagons from region $2\left(k=1.0\right.$ and $\left.E_{0}=0.56\right)$. Note in the second row that the spots in the far field become wider due to the linear growth of the unstable branch around $\vec{q}=0$ (Fig. 7).

the Goldstone modes, the neutrally stable eigenmodes due to the breaking of the translational invariance [40]. The Goldstone modes play a critical role in determining the near-field fluctuations of a Kerr hexagonal pattern in the presence of noise [41]. Figure 4 shows in detail the result of the stability analysis for a hexagonal pattern with $k=0.95$ for different values of the pump from below to above the instability border of region 1 (LW). The change of convexity of the soft mode branches clearly indicates the occurrence of a phase instability. Above the instability threshold, the saturation for large $q$ leads to a maximum growth rate at some finite values of $q$. This wave number can be clearly seen in the linear growth regime of numerical simulations.

Figure 5 shows a numerical simulation for $I_{s}=0.52$, taking as initial condition a stationary unstable hexagonal pattern with $k=0.95$, and shows the development of the instability. 

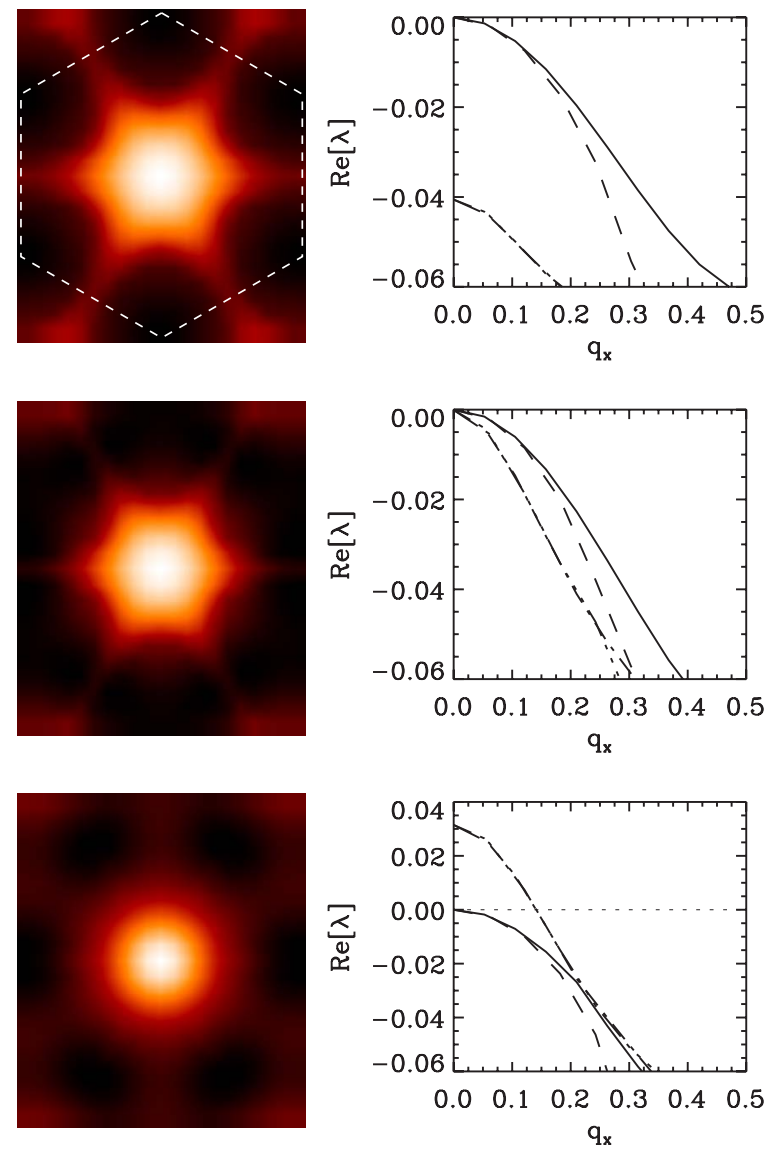

FIG. 10. (Color online) The same as in Fig. 4 for hexagons with a wave number $k=1.05$, crossing the upper border $(\mathrm{HO})$ of region 3 . From top to bottom, $I_{s}=0.51$ (stable), $I_{s}=0.52$ (critical point), and $I_{S}=0.53$ (Hopf unstable). In this case, the eigenvalues have a nonzero imaginary part.

In the first stages of the simulation the unstable wave vectors close to the wave vectors of the hexagonal pattern can be seen growing linearly (second row in Fig. 5). The Fourier components growing around the modes of the pattern in the far field must be compared with the prediction of the linear stability analysis in Fig. 4. Finally, the original hexagonal structure is replaced by another hexagonal structure with the same orientation but a larger wave number $(k=1.11)$. In finite systems, the minimum change in the wave number is imposed by the discrete Fourier space. In this case the pattern with the new wave number is not stationary. As shown in Fig. 3 for $I_{s}=0.52$ hexagons with this wave number are just above the HO line and therefore are unstable. The system finally evolves to a regime we call "chaotic hexagons," characterized by chaotically oscillating peaks on top of a regular hexagonal lattice [5].

The long-wavelength instability may take place also by decreasing the pump across the left border of region 1 (LW). As shown in Fig. 6, again an hexagonal pattern with the same orientation and a larger wave number (here $k \sim 1.0$ ) is formed. In this case the stationary pattern is stable.

\section{B. Amplitude instabilities}

Hexagonal patterns within regions 2 and 3 become unstable for perturbations with $\vec{q}=\overrightarrow{0}$ when the pump intensity is
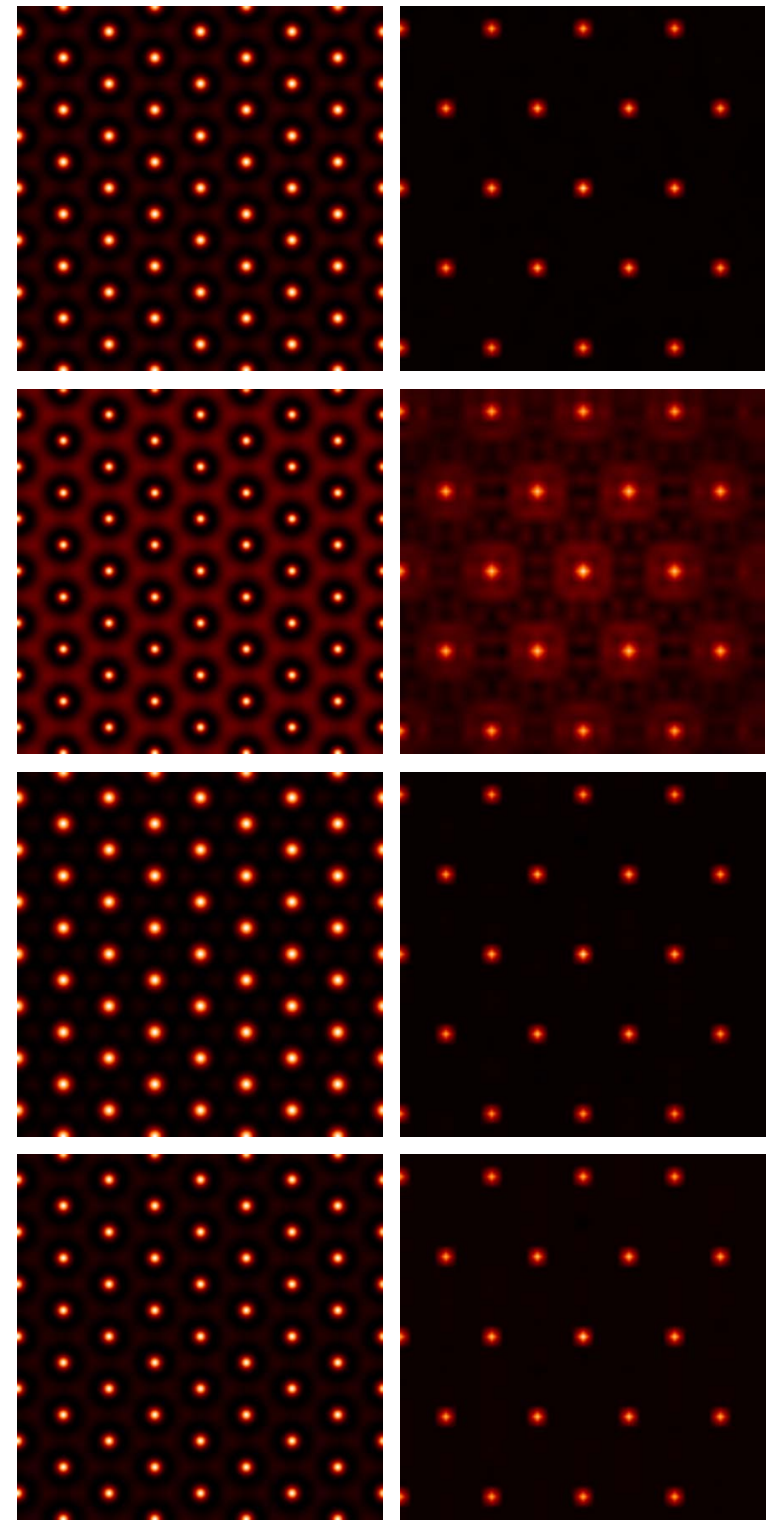

FIG. 11. (Color online) The same as in Fig. 5 for Hopf unstable hexagons from region $3\left(k=1.05\right.$ and $\left.I_{s}=0.52\right)$. The final hexagonal pattern oscillates homogeneously.

increased in such a way that $I_{S}$ goes above the instability lines $\mathrm{HS}$ and $\mathrm{HO}$. In region 2 the instability is stationary (HS), while in region 3 it is oscillatory (HO). These instabilities are associated to the growth of complex amplitude perturbations of the Fourier modes of the hexagonal pattern and, therefore, they are called amplitude instabilities. These instabilities are characterized by a parabolic branch $\lambda_{2}(\vec{q})$ centered at $\vec{q}=0$ and whose maximum $\lambda_{2}(\overrightarrow{0})$ goes from negative to zero, and then becomes positive. In fact, if we follow strictly the ordering of the eigenvalues, when it becomes positive, the branch $\lambda_{2}(\vec{q})$ should be called $\lambda_{0}(\vec{q})$, because it has crossed the two branches associated to the Goldstone modes.

\section{Stationary instability}

In this case $\lambda_{2}(\vec{q})$ is a branch of real eigenmodes, so, when $I_{S}$ is increased above the instability line HS the hex- 


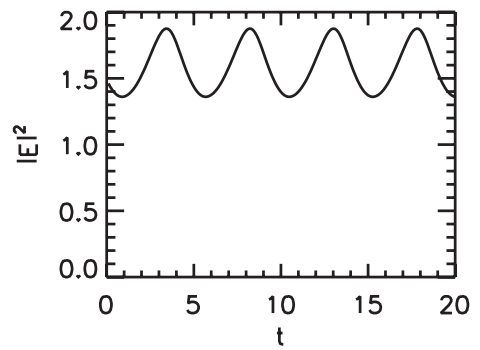

FIG. 12. Time evolution of the maximum of a hexagonal pattern peak in Fig. 11.

agonal patterns undergo a stationary bifurcation (Fig. 7). The unstable mode computed from the linear stability analysis is shown in Fig. 8. It has the same spatial wavelength that the pattern but the hexagonal structure in the near field is shifted a distance $l / \sqrt{3}$, where $l=2 \pi / k$ is the wavelength of the original pattern, in the direction forming a $30^{\circ}$ angle with the primary pattern.

Figure 9 shows a numerical simulation above the region 2 $\left(I_{s}=0.555\right)$ starting with an unstable stationary hexagonal pattern with $k=1.0$ as initial condition. In the transient (second and third rows of Fig. 9) one can glimpse the shape of the unstable mode (Fig. 8) predicted by the linear stability analysis as it grows. As a result, a new shifted hexagonal lattice appears in the near field. The interaction between the new peaks and those of the primary hexagonal pattern makes them oscillate, in a similar way as will be described in Sec. IV C for patterns with larger wave numbers. As a consequence spatial subharmonics are also generated. Finally, the system ends up in a spatiotemporal chaotic attractor that we call optical turbulence [5]. This regime is characterized by the formation of peaks at random positions that, after oscillating for several times with an overall broad temporal spectrum characteristic of chaotic behavior, vanish allowing other peaks to appear at nearby locations, thus evolving the whole pattern in a spatiotemporal chaotic manner. A further increase of the pump will destroy the spatial order and the system enters the optical turbulence regime [5].

\section{Oscillatory instability}

In region 3, when the pump is increased above the instability line $\mathrm{HO}$, the stationary hexagonal patterns undergo a homogeneous $(\vec{q}=0)$ Hopf bifurcation. In this case $\lambda_{2}(\vec{q})$ and $\lambda_{3}(\vec{q})$ are branches of complex conjugate eigenvalues. The real part of $\lambda_{2}(0)$ and $\lambda_{3}(0)$ become positive at the critical point with an imaginary part $\omega$ different from zero (Fig. 10). As a result, the hexagonal pattern starts to oscillate uniformly with a period $T=2 \pi / \omega$. Figure 11 shows the result of a simulation for $I_{s}=0.52$ starting from a Hopf unstable hexagonal pattern with $k=1.05$. Since the form of the unstable mode is similar to the stationary pattern, the hexagons simply start to oscillate. The period $T$ of the oscillation (Fig. 12) is in very good agreement with the value $T \simeq 4.5$ obtained from the imaginary part of the unstable eigenvalue of the stability analysis. The period of the oscillations increases with the pump, until, eventually, the uniformly oscillating hexagons become unstable and evolve towards chaotic hexagons.
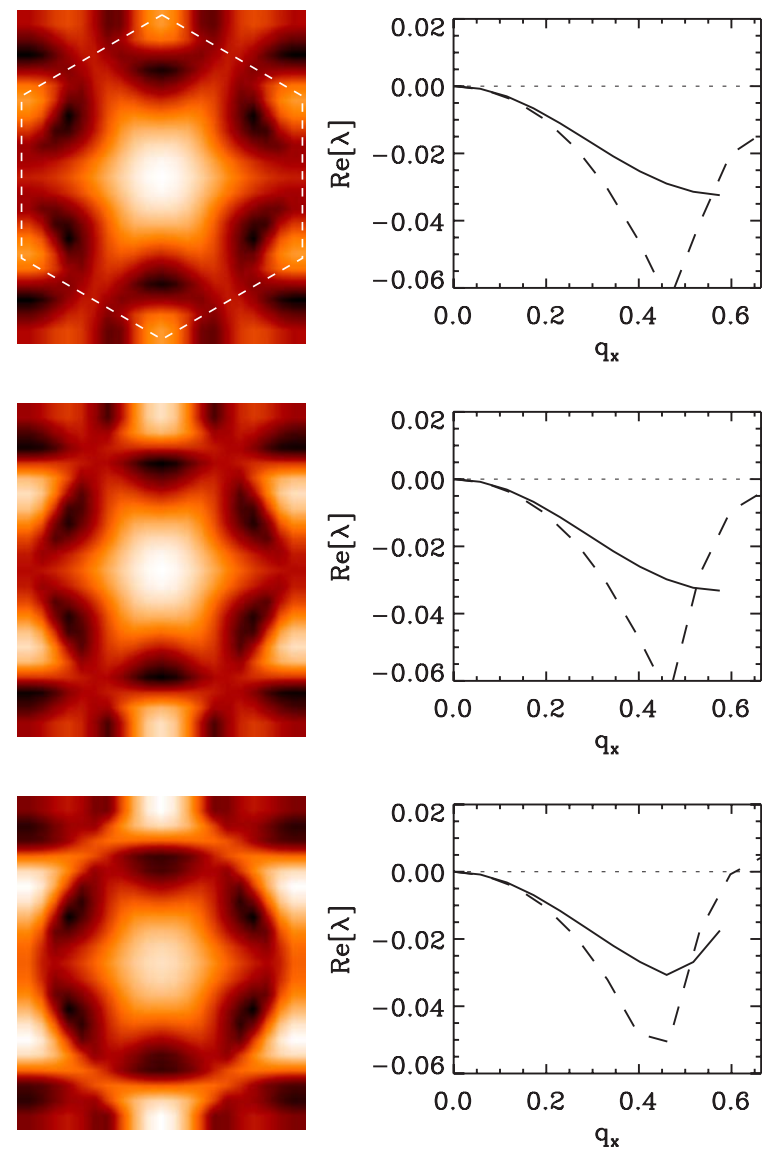

FIG. 13. (Color online) The same as in Fig. 4 for hexagons with a wave number $k=1.15$, crossing the upper border (FW) of region 4 . From top to bottom, $I_{s}=0.47$ (stable), $I_{s}=0.475$ (critical point), and $I_{s}=0.48$ (Hopf unstable).

\section{Finite wavelength Hopf instability}

In region 4 hexagonal patterns undergo a finite wavelength Hopf instability when the pump is increased above the instability line FW (Fig. 13). The unstable mode has a vector $\vec{q}_{1}=\left(\vec{k}_{1}^{0}+\vec{k}_{2}^{0}\right) / 3$, located on the vertex of the first Brillouin zone $\left(\left|\vec{q}_{1}\right|=k / \sqrt{3}\right)$ (Fig. 14). As a consequence hexagons do not oscillate uniformly, instead they are divided into three superlattices of wavelength $l_{1}=\sqrt{3} l$, corresponding to the spatial structure of the unstable eigenmodes (Fig. 14). Figure 15 shows the result of a numerical simulation for $I_{s}=0.48$ starting from an unstable hexagonal pattern with $k=1.15$.
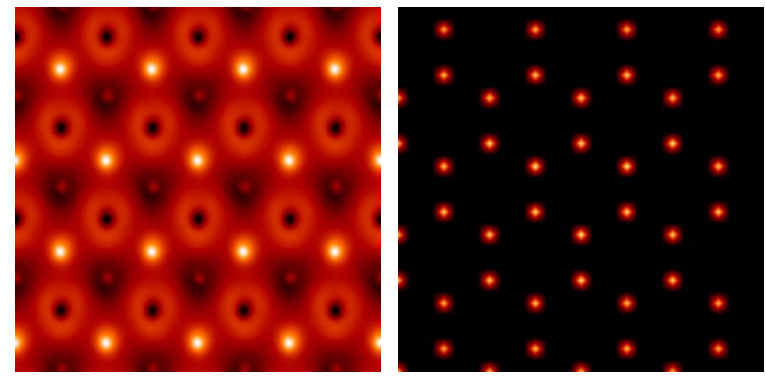

FIG. 14. (Color online) Near and far field of the mode then become unstable when the upper line of region $4(\mathrm{FW})$ is crossed. 

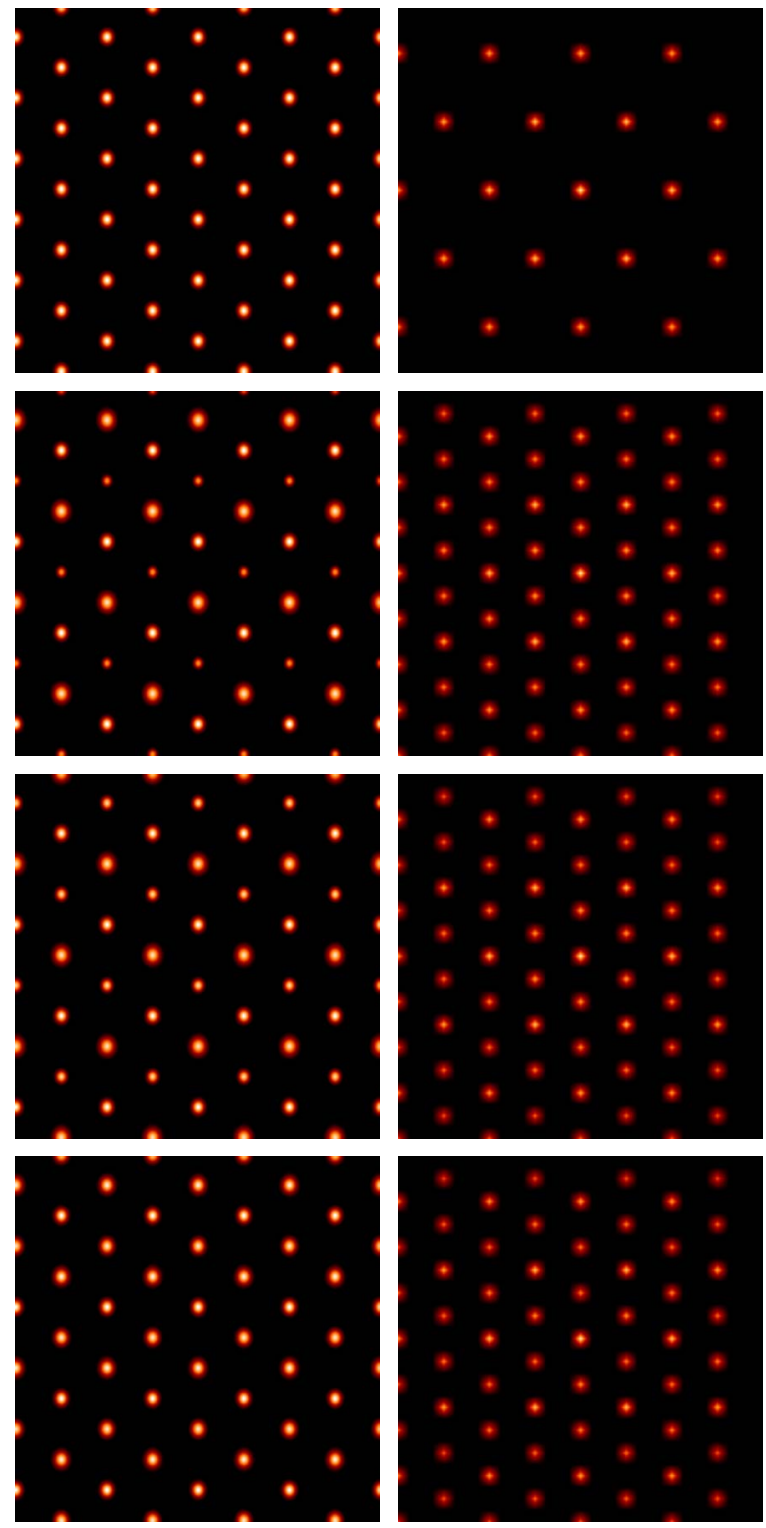

FIG. 15. (Color online) Time evolutions of $|E(\vec{x}, t)|^{2}$ (left) and $|E(\vec{k}, t)|^{2}$ (right) for Hopf unstable hexagons from region $4(k$ $=1.15$ and $\left.I_{s}=0.48\right)$. The hexagons do not oscillate homogeneously but nearby peaks are dephased. A superstructure corresponding to the unstable mode of Fig. 14 with a wave number $k / \sqrt{3}$ can be seen in the simulation.

The peaks in each superlattice oscillate synchronously. Two of the superlattices oscillate in antiphase with a relatively large amplitude while the third oscillates at twice that frequency and has a smaller amplitude. Figure 16 shows the time evolution of one of the peaks of each superlattice as well as the power spectrum corresponding to the time trace plotted with a solid line. As shown in the power spectrum, the frequency of the oscillations is in very good agreement with the value $\omega_{1}=\operatorname{Im}\left[\lambda_{0}\left(\vec{q}_{1}\right)\right] \simeq 1.0$ obtained from the linear stability analysis (vertical dashed line).

Physically this reflects an interchange of energy between two of the superlattices, mediated by the third while the total energy remains practically constant. Both in the homogeneous and finite wavelength Hopf instabilities, the high Fou-
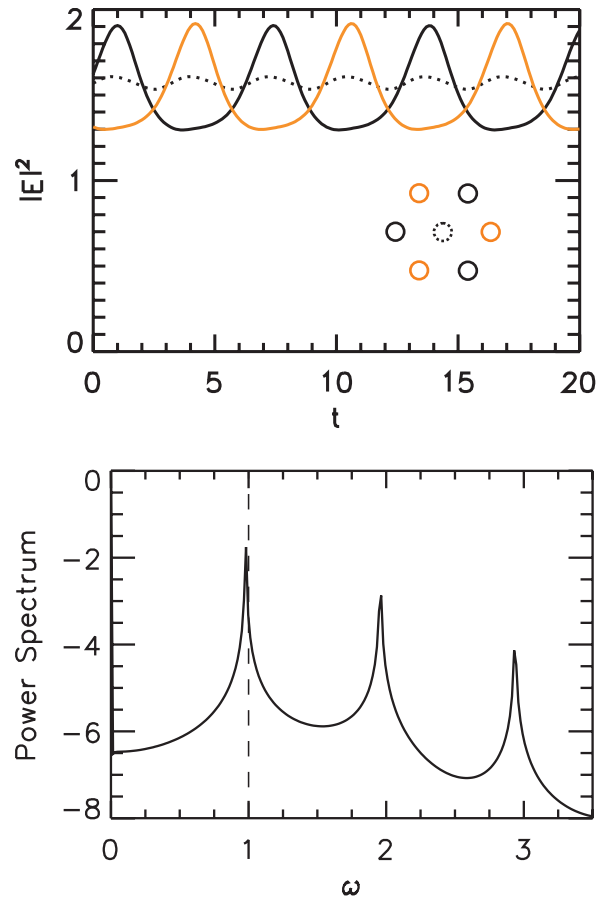

FIG. 16. (Color online) Left: Time evolution of the maximum of three dephased peaks of the hexagonal pattern in Fig. 15. Right: Power spectrum of the time trace plotted with a solid line on the left. The value of the frequency of the oscillations predicted by the stability analysis is indicated with a vertical dashed line.

rier harmonics play an important role in the oscillations. Neglecting or filtering out successive rings of high harmonics shift the bifurcations to larger pump values and may eventually suppress the bifurcations altogether [42-44]. Therefore the oscillations are associated to a transfer of energy from large scales to small scales. The bifurcation is supercritical, close to the critical point $I_{c}$ amplitude of the oscillations grows as $\sqrt{I_{s}-I_{c}}$ as shown in Fig. 17. Mathematically, this bifurcation is a spatial-period multiplying [23]. Stationary instabilities with the same wave vector $\vec{q}_{1}$ has been observed in surface waves [14] leading to superlattices.

A Bloch-Floquet analysis of the oscillatory hexagons reveal that increasing the pump intensity a subsequent Neimark-Sacker bifurcation further splits the the three superlattices, leading to a quasiperiodic regime in a route towards spatiotemporal chaos [5].

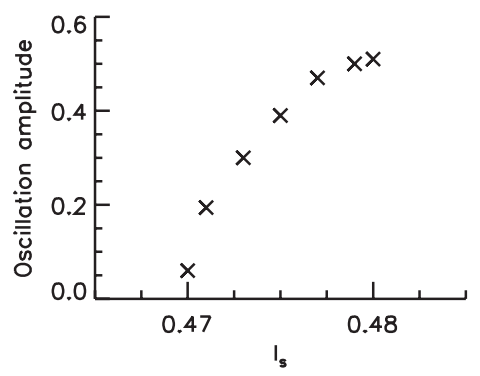

FIG. 17. Amplitude of the oscillations as a function of the control parameter $I_{s}$. 


\section{CONCLUDING REMARKS}

We have analyzed in detail the secondary bifurcations of stationary hexagonal patterns in a prototype model of nonlinear optics. The stability analysis of the stationary hexagonal patterns predicts different instabilities depending on their fundamental wave number. Hexagons with wave numbers smaller than the critical one undergo phase instabilities, patterns with wave numbers close to the critical one undergo stationary and oscillatory amplitude instabilities, and hexagons with larger wave numbers undergo finite wavelength Hopf instabilities leading to three superlattices oscillating periodically in antiphase. This instability can be also understood as a collective behavior of a hexagonal lattice of coupled oscillators. This system supports localized structures, also known as cavity or dissipative solitons, which can be considered as isolated peaks of a hexagonal pattern. These peaks also have an oscillatory instability $[6,7]$. The dynamics of this coherent structure is essentially two dimensional and, therefore, the hexagonal pattern can be interpreted as an hexagonal lattice of coupled oscillators. The coupling between two nearby peaks comes through diffraction. When the wave vector of the pattern is small (as it is the case for the patterns in region 3 ) the peaks are quite far away from each other, and the coupling is small. Then the system prefers to oscillate synchronously. Patterns in region 4 have a larger wave number and therefore a larger coupling between the peaks. In this circumstance a splitting in three superlattices is selected. In general, adding more energy to the system (increasing the pump) increases the influence of the coupling between peaks, so that uniformly synchronous oscillations become unstable (see Sec. IV B 2).

These results are typical and do not change significantly for small changes of the detuning parameter. Qualitative changes are observed for larger detunings, where the existence of localized structures may strongly modify the region of existence and stability of the hexagonal patterns.

\section{ACKNOWLEDGMENTS}

We acknowledge financial support from MEC (Spain), FEDER, and Govern de les Illes Balears Grants No. FIS2004-00953 (CONOCE2), No. TEC2006-10009 (PhoDeCC), and No. PROGECIB-5A.
[1] L. A. Lugiato and R. Lefever, Phys. Rev. Lett. 58, 2209 (1987).

[2] W. J. Firth, A. J. Scroggie, G. S. McDonald, and L. A. Lugiato, Phys. Rev. A 46, R3609 (1992).

[3] A. J. Scroggie, W. J. Firth, G. S. McDonald, M. Tlidi, R. Lefever, and L. A. Lugiato, Chaos, Solitons Fractals 4, 1323 (1994).

[4] W. J. Firth and G. K. Harkness, Asian J. Phys. 7, 665 (1998).

[5] D. Gomila and P. Colet, Phys. Rev. A 68, 011801(R) (2003).

[6] W. J. Firth et al., J. Opt. Soc. Am. B 19, 747 (2002).

[7] D. Gomila, M. A. Matias, and P. Colet, Phys. Rev. Lett. 94, 063905 (2005); D. Gomila, A. Jacobo, M. A. Matias, and P. Colet, Phys. Rev. E 75, 026217 (2007).

[8] M. Tlidi, R. Lefever, and P. Mandel, Quantum Semiclassic. Opt. 8, 931 (1996).

[9] E. Pampaloni, S. Residori, S. Soria, and F. T. Arecchi, Phys. Rev. Lett. 78, 1042 (1997).

[10] R. Herrero, E. Grosse Westhoff, A. Aumann, T. Ackemann, Yu. A. Logvin, and W. Lange, Phys. Rev. Lett. 82, 4627 (1999).

[11] T. Ackemann, E. Grosse Westhoff, M. Pesch, D. Rudolph, and W. Lange, Proc. SPIE 4751, 370 (2002).

[12] E. Große Westhoff, R. Herrero, T. Ackemann, and W. Lange, Phys. Rev. E 67, 025203 (2003).

[13] E. Große Westhoff, PhD thesis, Westfälische WilhelmsUniversität Münster, 2002 (unpublished).

[14] A. Kudrolli, B. Pier, and J. P. Gollub, Physica D 123, 99 (1998).

[15] C. Wagner, H. W. Müller, and K. Knorr, Phys. Rev. Lett. 83, 308 (1999).

[16] C. Wagner, H. W. Müller, and K. Knorr, Phys. Rev. E 62, R33 (2000).

[17] H.-J. Pi, S. Y. Park, J. Lee, and K. J. Lee, Phys. Rev. Lett. 84, 5316 (2000).
[18] J. L. Rogers, M. F. Schatz, O. Brausch, and W. Pesch, Phys. Rev. Lett. 85, 4281 (2000).

[19] W. Breazeal, K. M. Flynn, and E. G. Gwinn, Phys. Rev. E 52, 1503 (1995); A. L. Zanin, E. L. Gurevich, A. S. Moskalenko, H. U. Bödeker, and H.-G. Purwins, ibid. 70, 036202 (2004).

[20] B. Dionne and M. Golubitsky, ZAMP 43, 36 (1992).

[21] B. Dionne, M. Silber, and A. C. Skeldon, Nonlinearity 10, 321 (1997).

[22] M. Silber and M. R. E. Proctor, Phys. Rev. Lett. 81, 2450 (1998).

[23] D. P. Tse, A. M. Rucklidge, R. B. Hoyle, and M. Silber, Physica D 146, 367 (2000).

[24] S. L. Judd and M. Silber, Physica D 136, 45 (2000).

[25] P. Coullet and G. Iooss, Phys. Rev. Lett.. 64, 866 (1990).

[26] L. Gil, Physica D 147, 300 (2000).

[27] C. Pirat and L. Gil, Physica D 179, 92 (2003).

[28] A. C. Newell and J. Moloney, Nonlinear Optics (AddisonWesley, Redwood City, 1992).

[29] C. Sulem and P.-L. Sulem, The Nonlinear Schrodinger Equation: Self-Focusing and Wave Collapse (Springer-Verlag, New York, 1999).

[30] R. Fedele and H. Shamel, Eur. Phys. J. B 27, 313 (2002).

[31] P. K. Shukla, N. N. Rao, M. Y. Yu, and N. L. Tsindsadze, Phys. Rep. 138, 1 (1986).

[32] D. Gomila, T. Ackemann, E. G. Westhoff, P. Colet, and W. Lange, Phys. Rev. E 69, 036205 (2004).

[33] G. K. Harkness, W. J. Firth, G.-L. Oppo, and J. M. McSloy, Phys. Rev. E 66, 046605 (2002).

[34] Y. A. Kuznetsov, Elements of Applied Bifurcation Theory (Springer-Verlag, New York, 1995).

[35] G. K. Harkness, R. Martin, A. J. Scroggie, G.-L. Oppo, and W. J. Firth, Phys. Rev. A 58, 2577 (1998).

[36] M. Le Berre, A. S. Patrascu, E. Ressayre, and A. Tallet, Phys. 
Rev. A 56, 3150 (1997).

[37] D. Walgraef, Spatio-Temporal Pattern Formation (SpringerVerlag, New York, 1997).

[38] J. Lauzeral, D. Metens, and D. Walgraef, Europhys. Lett. 24, 707 (1993).

[39] M. Bestehorn, Phys. Rev. E 48, 3622 (1993).

[40] M. M. Sushchik and L. S. Tsimring, Physica D 74, 90 (1994).

[41] B. Echebarria and C. Pérez-García, Europhys. Lett. 43, 35
(1998).

[42] D. Foster, Hydrodynamic Fluctuations, Broken Symmetry and Correlation Fluctuations (Addison Wesley, Redwood City, CA, 1983).

[43] D. Gomila and P. Colet, Phys. Rev. E 66, 046223 (2002).

[44] When performing the linear stability analysis we explore values of $\vec{q}$ with increments $\Delta q_{x}=k / 20$ and $\Delta q_{y}=k \sin \left(60^{\circ}\right) / 20$. 